\title{
Abstracts From the 6th Annual Congress on Medicine \& Science in Ultra-Endurance Sports, October 11-13, 2019, Cape Town, South Africa
}

\section{Introduction}

The 6th Annual Congress on Medicine \& Science in Ultra-Endurance Sports will be held October 11-13, 2019, in Cape Town, South Africa, in conjunction with the South African Sports Medicine Association Conference 2019. Details of this Congress, as well as past and future meetings, can be found at the Ultra Sports Science Foundation website: http:// ultrasportsscience.us.

Ultra-endurance activities remain fertile ground for research since there are many unanswered questions directly related to these activities, and ultra-endurance activities can serve as viable models for other physiological or psychological stress. The Ultra Sports Science Foundation is pleased to be able to support some of this research through our grantfunding programs and the sharing of research findings at our annual international conference

\section{Martin D. Hoffman, Sacramento VA Medical Center,} Founding Member Ultra Sports Science Foundation

Participant Opinions and Expectations About Medical Services at Ultramarathons: Findings From the ULTRA Study

Martin D. Hoffman, MD

VA Northern California Health Care System, University of California Davis Medical Center, and Ultra Sports Science Foundation

Objective.-This work explores the opinions and expectations of ultramarathon runners about medical services and their perceived quality during ultramarathons. Methods.-Focused questions related to medical services at ultramarathons were included in the 2018 survey of Ultrarunners Longitudinal TRAcking (ULTRA) Study enrollees. Results.-Among the 1135-1156 respondents, $83.2 \%$ agreed that ultramarathons should provide at least a minimum level of medical support with basic first aid and emergency transport services rated as the most important medical services, and individuals with basic first aid training rated as the most important medical providers at ultramarathons. Participant safety was felt to largely be the responsibility of each runner as well as the race and/or medical director. Among 832 respondents having completed an ultramarathon in 2016-2018, their impression of medical services at 4853 ultramarathons was generally favorable. Of the $4.0 \%$ of times in which medical support was needed, it met expectations $74.0 \%$ of the time. Of the total of 240 different medical issues for which medical support was needed, blister management was the most common, accounting for $26.7 \%$ of issues. Conclusions.-Even though medical services receive minimal utilization during ultramarathons, ultramarathon runners largely believe that these events should provide at least a minimum level of medical support.
Ultramarathon runners place a high onus for safety during ultramarathons on themselves, but they also place a high level of responsibility on race and medical directors, so it seems prudent for races to avoid a mismatch between runner expectations and the medical services actually provided.

Changes in Urinary Markers of Acute Kidney Injury After 10 km and 100 km Races

Wojciech Ratkowski, $\mathrm{PhD}^{1}$; Wojciech Wołyniec, $\mathrm{PhD}^{2}$; Katarzyna Zorena, $\mathrm{PhD}^{2}$; Katarzyna Kasprowicz, $\mathrm{PhD}^{1}$; Maria Bartoszewicz, $\mathrm{PhD}^{2}$; Marcin Renke, $\mathrm{PhD}^{2}$

${ }^{1}$ Gdansk University of Physical Education and Sport, ${ }^{2}$ Medical University of Gdansk, Poland

Objective.-Acute kidney injury (AKI) is described as a relatively common complication of strenuous exercise. In clinical practice the diagnosis of AKI is based on increase of creatinine level. Serum creatinine rises about 24-48 hours after renal injury and is dependent on age, sex, muscle mass, diet and hydration status. Therefore after physical exercise diagnosis of AKI based on creatinine is overestimated. There are several urinary makers of AKI: albuminuria (uAlb), neutrophil gelatinase-associated lipocalin (NGAL), kidney injury molecule 1 (KIM-1) and cystatin $\mathrm{C}(\mathrm{CyC})$, which are used to diagnose early kidney dysfunction. Methods. -16 runners, mean age 37 \pm 8 .1years participating in 100 and $10 \mathrm{~km}$ races were studied. Before and after the races blood and urine were taken to assess markers of AKI. Creatinine was measured in blood. Albumin, NGAL KIM-1 and CyC were examined in urine. Results.-All urinary makers of AKI and creatinine increased significantly after both races. There was no correlation between changes in creatinine and any of urinary markers of AKI.

\begin{tabular}{llllll}
\hline & \multicolumn{2}{c}{ 10 km race } & & \multicolumn{2}{c}{100 race } \\
\cline { 2 - 3 } \cline { 5 - 5 } & \multicolumn{1}{c}{ Before } & \multicolumn{1}{c}{ After } & & Before & \multicolumn{1}{c}{ After } \\
\hline $\begin{array}{l}\text { Creatinine } \\
(\mathrm{mg} / \mathrm{dl})\end{array}$ & $0.78 \pm 0.14$ & $0.99 \pm 0.18^{*}$ & $0.85 \pm 0.13$ & $1.2 \pm 0.27^{*}$ \\
$\mathrm{uAlb}(\mathrm{mg} / \mathrm{l})$ & $5.7(5-7.57)$ & $\begin{array}{l}40.67 \\
(15.7-126.3)^{*}\end{array}$ & & $3(3-9.55)$ & 57.15 \\
& & $19(9-30)^{*}$ & $4.4(2.15-6)$ & $30(19-63)^{*}$ \\
$\mathrm{uNGAL}$ & 4.9 & & & \\
$(\mathrm{ng} / \mathrm{ml})$ & $(0.85-8.3)$ & & & \\
$\mathrm{uKIM}-1$ & 0.44 & $1.29(0.19-$ & 0.21 & 1.63 \\
$(\mathrm{ng} / \mathrm{ml})$ & $(0.15-1.11)$ & $2.47)^{*}$ & $(0.08-0.78)$ & $(0.58-2.49)^{*}$ \\
$\mathrm{uCyC}(\mathrm{ng} /$ & 45.95 & $117.7(71.95-$ & 28.28 & 138.5 \\
$\mathrm{ml})$ & $(5.7-89.95)$ & $144.5)^{*}$ & $(2.4-88.5)$ & $(115.9-155.6)^{*}$ \\
\hline
\end{tabular}

$* \mathrm{p}<0.05$, Data were presented as mean $\pm \mathrm{SD}$, or median (IQR) 
Conclusions.-All urinary markers of AKI increased after long runs. These changes were dependent on race duration. The highest increase was noticed in the albuminuria and UNGAL. It is not clear if AKI markers can help to differentiate between functional and structural kidney injury.

Pelvic Fracture and Low Bone Density in a Long Distance Cyclist With a History of Nonclassic Congenital Adrenal Hyperplasia

Karin VanBaak, MD

University of Colorado School of Medicine, USA

Background.-Nonclassic congenital adrenal hyperplasia (NCAH) is an autosomal recessive disorder which results in impaired cortisol biosynthesis and androgen excess. There is limited data on bone mineral density (BMD) outcomes in patients with NCAH. Case Report.-A 24 year-old female long distance cyclist presented for evaluation of pelvic pain two days after a low velocity bike crash. Her exam showed tenderness to palpation of the left pubic ramus, a positive single leg hop test and normal gait. X-ray revealed a nondisplaced fracture of the left inferior pubic ramus. MRI revealed an additional nondisplaced fracture of the ipsilateral sacral ala. Her medical history was pertinent for $\mathrm{NCAH}$, which was diagnosed in her late teenage years during a workup for primary amenorrhea. She had never been treated, either with glucocorticoids or exogenous estrogen. She had a self-limited history of disordered eating during adolescence. Her current activity consisted of long distance multi-day bike-pack trips, racing road bikes, and distance running. She had a progesterone IUD but used no other medications. Dual energy x-ray absorptiometry scan showed low bone density with L1-4 Z-score -1.8. Serum calcium and Vitamin D were within normal limits. She was treated for her fractures and returned to activity. Discussion regarding her endocrine management plan was put on hold when she left for a 6 month bike-pack trip through South America. Conclusions.-Unexpected fractures in endurance athletes should prompt evaluation for underlying medical conditions. While low energy availability, secondary amenorrhea, and low bone density are common in endurance athletes, rare medical conditions should also be considered. We report a case of a long distance cyclist with a history of $\mathrm{NCAH}$, no past treatment with glucocorticoids, and past low energy availability who presented with a pathologic fracture and low bone density.

The Impact of Post-Exercise Chocolate Flavoured Dairy Milk Beverage Consumption on Hydration Status in Endurance Athletes

Isabella Russo, MNutr ${ }^{1}$; Alan McCubbin, BNutr ${ }^{1}$; Judi Porter, $\mathrm{PhD}^{1,2}$; Louise Burke, $\mathrm{PhD}^{3}$; Ricardo J.S Costa, $\mathrm{PhD}^{1}$

${ }^{1}$ Monash University, Department of Nutrition \& Dietetics, Notting Hill, Victoria, Australia, ${ }^{2}$ Allied Health Clinical Research Office, Eastern Health, Box Hill, Victoria, Australia, ${ }^{3}$ Department of Sports Nutrition, Australian Institute of Sport, Bruce, ACT, Australia

Objective.-The study aimed to comprehensively determine the impact of consuming a dairy milk beverage after prolonged strenuous exercise on hydration status. Methods.-Using a randomised crossover study design, 11 endurance male athletes consumed a chocolate flavoured dairy milk beverage (CM: $1.2 \mathrm{~g} / \mathrm{kg}$ carbohydrate and $0.4 \mathrm{~g} / \mathrm{kg}$ protein) or a carbohydrate-electrolyte beverage (CEB: isovolumetric with $0.76 \mathrm{~g} / \mathrm{kg}$ carbohydrate intake) after completing a $2 \mathrm{~h}$ glycogen depleting exercise protocol in temperate ambient conditions $\left(20-25^{\circ} \mathrm{C}, 40 \% \mathrm{RH}\right)$. The recovery beverage was provided in three equal boluses over a $30 \mathrm{~min}$ period. Throughout the remainder of the $4 \mathrm{~h}$ recovery period, participants were provided with water, towards a total fluid intake targeting $35 \mathrm{ml} / \mathrm{kg}$. Venous blood samples were collected, total body water (MBIA) and nude body mass were measured pre-exercise and throughout recovery. Whole blood samples were used to measure haemoglobin concentration and haematocrit to determine $\Delta \mathrm{P}_{\mathrm{V}}$, while $50 \mu$ l of heparin plasma was used to determine $\mathrm{P}_{\mathrm{Osmol}}$ by freeze-point osmometry. Results.-Exerciseinduced body mass loss (overall mean (SD): 1.4 (0.6) \%; p=0.704), $\left.\Delta \mathrm{P}_{\mathrm{V}}(-1.5(2.6) \%) ; \mathrm{p}=0.187\right)$, and pre- to post-exercise $\mathrm{P}_{\text {Osmol }}(293$ (6) to 295 (7) $\mathrm{mOsmol} / \mathrm{kg}$, respectively; $\mathrm{p}=0.297$ ) did not differ between CM and CEB. During the recovery period, there was a main effect of time imposed by the rehydration intervention for $\mathrm{P}_{\mathrm{Osmol}}(\mathrm{p}=0.008), \Delta \mathrm{P}_{\mathrm{V}}$ $(\mathrm{p}<0.001)$, and rating of thirst $(\mathrm{p}<0.001)$, but not total body water $(\mathrm{p}=0.830)$. No trial differences were observed. Total fluid intake did not differ between trials (CM: $24.2 \mathrm{ml} / \mathrm{kg}$ vs CEB: $24.0 \mathrm{ml} / \mathrm{kg}$; $\mathrm{p}=0.907$ ). However, there was greater fluid retention (CM: $85.8 \%$ vs CEB: $76.1 \%$; $\mathrm{p}=0.021$ ) with a corresponding lower urine output (CM: 248ml; CEB: 409ml; $\mathrm{p}=0.008$ ) on CM. Conclusions. - Both CM and CEB resulted in complete rehydration during recovery from prolonged strenuous exercise. However, greater fluid retention was observed on CM, suggesting a superior rehydration fluid option over CEB.

Supported by Lion Dairy \& Drinks Co.

Does Training Status and Protein Intake Explain the Differences in Physical Performance Between Trained Endurance and Recreationally Active Older Adult Males?

Zoya Huschtscha, APD; Judi Porter, PhD; Ricardo J.S. Costa, PhD

Monash University, Department of Nutrition Dietetics \& Food, Notting Hill, Victoria, Australia

Objective.-The study aimed to examine whether differences in prerequisites to sarcopenia (i.e., skeletal muscle mass, strength and physical performance) exist between endurance trained (ET) and recreationally active (RA) older adults; and whether training status, age and protein intake have intended effects on these outcomes. Methods.-Using preliminary data from a larger clinical trial $n=19($ ET $n=9$, RA $n=10)$ older active adult males: (mean (SD) 62.5 (6.3) years, 78.07 (12.6) kg, 176 (7.6) cm, 27.7 (7.6) \% body fat) that exercised $\geq 3 x /$ week (217 (13) $\mathrm{min} /$ week) volunteered to participate in the baseline measurements. Body composition was determined by iDXA (GE LUNAR iDXA NarrowAngle Dual Energy X-ray Densitometer), and strength by handgrip (dynamometer). Performance measures were determined by jump height (400S Force Plate with the XPV7 Diagnostic Test Software) and cardiorespiratory fitness (CRF) through an incremental bike test (Corival, InMed). PA and nutritional intake were determined by PAR-Q and 3-day food-fluid diary, respectively. Data were analysed (SPSS v25.0) for group differences and associations (Pearson's correlation coefficient) between strength and performance outcomes with protein intake and age. Results.-Significant between group differences were observed for lean body mass (ET: 60.4 (6.4) kg, RA: 53 (8.5) kg; p<0.05), jump height (ET: 19.7 (4.7) cm, RA: 15.6 (3.5) cm; p<0.05), and CRF (ET: 152.1 (32.06) watts/RQ, RA: 80 (36.1) watts/RQ; $\mathrm{p}<0.05)$. There was no significant group difference for handgrip (ET: 45.6 (7.59) kg, RA: 41.5 (4.2) kg; p=0.116). There were no significant correlations between age, protein intake, and weekly exercise volume with anthropometry, metabolic, strength and performance variables. However, higher levels of exercise intensity were positively correlated with higher lean body mass ( $r=0.452, \mathrm{p}=0.05)$, CRF $(\mathrm{r}=0.723, \mathrm{p}<0.05)$, but not jump height or handgrip. Conclusions.-ET presents favourable outcomes of lean body mass, CRF, and performance in comparison to RA. Exercise intensity appears to prompt these outcomes, suggesting ET may mitigate some of the sarcopenic effects associated with ageing.

Supported by Lion Dairy \& Drinks Co. 
The Value of Lactate Thresholds in Performance Prediction in Trail Running

Volker Scheer, $\mathrm{MD}^{1,2,3}$; Solveig Vieluf, $\mathrm{PhD}^{3}$; Tanja I Janssen, $\mathrm{Msc}^{3,4}$; Hans-Christian Heitkamp, $\mathrm{MD}^{3}$

${ }^{1}$ Ultra Sports Science Foundation, Pierre Benite, France, ${ }^{2}$ Universidad a distancia de Madrid (UDIMA), University of Madrid, Spain, ${ }^{3}$ Institute of Sports Medicine, Department of Health, University of Paderborn, Germany, ${ }^{4}$ Institute of Sports Science, University of Münster, Germany

Objective.-Trail running is a growing and popular sport, held in natural environments with less than $20 \%$ on paved roads, as defined by its governing body, the International Trail Running Association (ITRA). Performance prediction and improving competition results are important. Lactate thresholds (LT) are a classical performance predictor in road running but to date little is known of its value in trail running. Our aim was to evaluate established LT concepts in a group of highly trained trail runners examining their predictive character to a popular trail race competition. Methods.Twenty five trail runners (mean age $31.2 \pm 5.1$ years) completed a standard graded exercise test on the treadmill for determination of maximal oxygen uptake $\left(\mathrm{VO}_{2} \max 59.5 \pm 5.2 \mathrm{ml} \cdot \mathrm{kg}^{-1} \mathrm{~min}^{-1}\right)$ and LT. Values and velocities for aerobic LT (AET), individual anaerobic LT (IAT according to Dickhuth) and fixed LT at $4 \mathrm{mmol} / \mathrm{l}$ (LT4) were obtained. All runners participated subsequently in an official $31.1 \mathrm{~km}$ trail running race (positive/negative elevation $515 \mathrm{~m} / 710 \mathrm{~m}$; XS classification according to ITRA). Correlation analysis was performed to predict the value of the race results. Statistical significance was set at $\mathrm{p}<0.05$. Results. - Values obtained for the different LT were: AET $(1.03 \pm 0.59 \mathrm{mmol} / \mathrm{l} ; 11.2 \mathrm{~km} / \mathrm{h} \pm 1.1)$, IAT $(2.53 \pm 0.59 \mathrm{mmol} / \mathrm{l} ; 15.4 \pm 1.6 \mathrm{~km} / \mathrm{h})$ and LT4 $(16.2 \pm 1.9 \mathrm{~km} / \mathrm{h})$. Mean race times for the XS trail race were $02 \mathrm{~h} 12 \mathrm{~min} 24 \mathrm{~s} \pm 00 \mathrm{~h} 12 \mathrm{~min} 01 \mathrm{~s}$. Race performance times correlated with AET: $r=-0.65, p<0.01$; LT4: $r=-0.87, p<0.01$; IAT: $r=-0.84, p<0.01$ ), meaning the higher the speed at the LT the faster the race performance. Conclusions.XS trail race performance time correlated with the fixed $4 \mathrm{mmol} / \mathrm{l} \mathrm{LT}$ and the individual LT according to Dickhuth. LT can be of value in predicting trail running race performance and aid in designing training plans.

Can Electromyographic Threshold and Critical Speed Overestimate the Lactate Threshold in Marathon and Ultramarathon Runners?

Jeferson M. Vianna, $\mathrm{PhD}^{1}$; Yuri A. C. Campos, $\mathrm{MS}^{1,2}$; Miller P. Guimarães, $\mathrm{MS}^{2,3}$; Raúl D. Herrera, $\mathrm{PhD}^{4}$; Sandro F. Silva, $\mathrm{PhD}^{2}$; Jefferson S. Novaes, $\mathrm{PhD}^{1,5}$; Victor M. Reis, $\mathrm{PhD}^{6}$

${ }^{1}$ Postgraduate Program of the Faculty of Physical Education and Sports of the University of Juiz de Fora, ${ }^{2}$ Study Group and Research in Neuromuscular Responses, University of Lavras, ${ }^{3}$ Postgraduate in Human Movement and Rehabilitation Sciences, University of São Paulo, ${ }^{4}$ Faculty of Health Sciences, Isabel I University, ${ }^{5}$ Postgraduate Program of Physical Education of the University of Rio de Janeiro, ${ }^{6}$ Center for Research in Sports Sciences, Health Sciences and Human Development, University of Trás-os-Montes and Alto Douro

Objective.-The objectives of the study were to determine the running speed at lactate threshold (LT), electromyographic fatigue threshold in the vastus lateralis (EMGFTVL), and critical speed (CS) and to verify if there is a correlation among these exercise intensity domains. Methods. - Twelve male marathon and ultra-marathon runners (age $=$ $24.6 \pm 4.9$ years; best time in $5,000 \mathrm{~m}=21 \mathrm{~min} 22 \mathrm{sec} \pm 1 \mathrm{~min} 39 \mathrm{sec}$; training experience $=4.4 \pm 1.3$ years) volunteered to participate in the study. For data collection, the participants were randomized into three separate test sessions in a 48-hour interval. In the first session, the participants performed an incremental treadmill protocol where the blood lactate [LT - by Dmax method] and electromyographic signal amplitude
[EMGFTVL - by De Vries method] were collected. In the second and third sessions, the participants performed time-trials at distances of 3,000 and $5,000 \mathrm{~m}$ on an outdoor running track for the calculation of the CS through the linear regression method. Results.-Our results showed that LT, EMGFTVL and CS were found at running speeds of $13.48 \pm 1.25,14.72 \pm 1.14$, and $14.48 \pm 0.35 \mathrm{~km} . \mathrm{h}^{-1}$, respectively. There were significant differences between LT and EMGFTVL $(p=.002)$, and between LT and CS $(p=.010)$. However, there was no difference between EMGFTVL and CS ( $p=.956)$. In addition, correlations between $\mathrm{LT}$ and EMGFTVL $[\mathrm{r}=.74 / \mathrm{ICC}=.672], \mathrm{LT}$ and $\mathrm{CS}[\mathrm{r}=.77 / \mathrm{ICC}=.764]$, and EMGFTVL and CS [r=.84/ICC $=.901]$ were found. Conclusions.The results of the present investigation demonstrate that the EMGFTVL and CS are determined at statically different running speeds in relation to the LT. These findings indicated that LT can be used to mark the transition between the moderate and heavy domains, while the EMGFTVL and CS can be used to mark the transition between the heavy and severe domains.

The Impact of Ultra-Formulated Dehydrated Meal Consumption Along a Multi-Stage Utramarathon Competition on Gastrointestinal Symptoms and Nutritional Status of Ultra-Endurance Runners: A Double-Blind Randomised Control Trial

Ricardo J.S. Costa, $\mathrm{PhD}^{1}$; Alexandra Stone, BNutr ${ }^{1}$; Alice Mika, BNutr ${ }^{1}$; David Barnett, $\mathrm{PhD}^{2}$; Vera Camoes-Costa, $\mathrm{PhD}^{1}$

${ }^{I}$ Monash University, Department of Nutrition Dietetics \& Food, Notting Hill, Victoria, Australia, ${ }^{2}$ Australian Catholic University, Institute for Health \& Aging, Melbourne, Victoria, Australia

Objective.-Gastrointestinal symptoms (GIS) are common in ultramarathon events, contributing to decrements in nutritional status, with potency to impact physical performance. The study aimed to determine the impact of ultra-formulated dehydrated meal consumption on GIS and nutritional status of ultra-endurance runners (UER) along a $136 \mathrm{~km}$ four-stage multistage ultramarathon (MSUM; Run Larapinta, Alice Springs, Australia). Methods.-Fourteen UER were randomly allocated into an in-house formulated (BASE; $n=8$ ) or commercially available $(\mathrm{CA} ; \mathrm{n}=6)$ dehydrated/freeze-dried meal group. In addition to meal provisions, participant consumed foods and fluids ad libitum, which was recorded in a food-fluid intake log and analysed by dietary analysis software. Estimated energy expenditure was determined by a validated tri-axial accelerometer. Body mass and body water variables (MBIA) were measured before and on completion of the MSUM. GIS incidence and severity during running and recovery were determined using a modified-VAS. Results.-Estimated energy expenditure was (overall mean \pm SD) $4832 \pm 972 \mathrm{kcal} /$ day. Total energy and macronutrient $(2410 \pm 869 \mathrm{kcal} / \mathrm{day}$, protein $12 \%$, carbohydrate $62 \%$, and fat $26 \% ; \mathrm{p}=0.039)$, but not water $(60.4 \pm 32.1 \mathrm{ml} / \mathrm{kg} / \mathrm{day}$; $\mathrm{p}=0.364$ ), intakes were observed to be higher on BASE compared with CA. $\Delta$ pre- to post-MSUM body mass was $-3.5 \pm 2.7 \%$ and $-4.0 \pm 2.3 \%$, $\Delta$ body fat mass was $-2.7 \pm 2.7 \%$ and $-1.8 \pm 1.4 \%$, and $\Delta$ fat-free mass dry weight was $-0.4 \pm 0.8 \mathrm{~kg}$ and $-0.8 \pm 0.7 \mathrm{~kg}$ on BASE and CA, respectively $(\mathrm{p}>0.05)$. Total body water increased on BASE $(0.5 \pm 2.2 \mathrm{~L})$ and decreased on CA $(-0.5 \pm 1.4 \mathrm{~L})$ pre- to post-MSUM ( $>0.05)$. GIS incidence was reported by $86 \%$ of UER along the MSUM. A reduction in total-GIS severity was observed on BASE $(\mathrm{p}=0.050)$ during running and recovery, but not on CA. Conclusion.-Despite energy and nutritional requirements observed to be sub-optimal in all UER in response to ad libitum food and fluid intake during MSUM, the provisions of BASE resulted in a more favourable nutritional and hydration profile, in comparison with CA.

Supported by the Technologies Australia Sports Company 
Does the Gut Microbiota Bacterial Abundance and Composition Influence the Magnitude of Intestinal Integrity Perturbations, Systemic Cytokinaemia, and Gastrointestinal Symptoms in Response to Exertional-Heat Stress?

Christie Bennett, BNutr Hon ${ }^{1}$; Rebekah Henry, $\mathrm{PhD}^{2}$; Rhiannon M.J. Snipe, $\mathrm{PhD}^{3}$; Ricardo J.S. Costa, $\mathrm{PhD}^{1}$

${ }^{1}$ Monash University, Department of Nutrition Dietetics \& Food, Notting Hill, Victoria, Australia, ${ }^{2}$ Monash University, Department of Civil Engineering-Microbiology, Clayton, Victoria, Australia, ${ }^{3}$ Deakin University, Centre of Sport Research, Burwood, Victoria, Australia

Objective.-Emerging evidence suggests the gastrointestinal commensal and pathogenic bacteria may potentially influence gastrointestinal perturbations in response to exertional-heat stress, with potential exercise performance and health implications. The study aimed to examine if the relative bacterial abundance and diversity of the intestinal microbiota influences the magnitude of markers characteristic of exercise-induced gastrointestinal syndrome (EIGS). Methods.-Twenty-two endurance-trained athletes ( $\mathrm{n}=13$ males and $\mathrm{n}=9$ females) completed $2 \mathrm{~h}$ running at $60 \% \mathrm{VO}_{2} \max$ in $35.2 \pm 1.2^{\circ} \mathrm{C}(26 \pm 3 \%$ $\mathrm{RH})$. A mid-flow faecal sample $(\sim 30 \mathrm{~g})$ was collected pre-exercise, and immediately stored at $-80^{\circ} \mathrm{C}$ before analysis. Faecal microbiota taxonomy was determined by next generation sequencing (Illumina MiSeq platform). Data were processed using the QIIME2 pipeline (v2019.1) establishing taxonomic classification with $>95 \%$ confidence using SILVA. Blood samples were collected pre- and post-exercise to determine I-FABP, cortisol, and cytokine profiles. Markers of physiological and thermoregulatory strain, and gastrointestinal symptoms (GIS) were measured throughout. Correlations and $\beta$-diversity analysis between amplicon sequence variants (ASVs) and EIGS markers were performed. Results. - Bacterial phyla $(n=11)$, family $(n=42)$, and genus $(n=159)$ ASVs were detected for relative abundance and $\alpha$-diversity (Shannon index) determination. Significant correlations were observed between I-FABP and relative abundance of pathogenic Tenericutes $\left(r_{s}=0.438, \mathrm{p}=0.041\right)$, and phlya $\alpha$-diversity $\left(r_{s}=-0.608, \mathrm{p}=0.012\right)$ with cytokinaemia. Significant correlations were observed between I-FABP and relative abundance of Flavobacteriaceae $\left(r_{s}=0.450, \mathrm{p}=0.036\right)$, Peptococcaceae ( $\left.r_{s}=0.450, \mathrm{p}=0.036\right)$, Ruminococcaceae $\left(r_{s}=0.498, \mathrm{p}=0.019\right)$, and Barnesiellaceae $\left(r_{s}=-0.540, \mathrm{p}=0.031\right)$ and family $\alpha$-diversity $\left(r_{s}=-0.732, \mathrm{p}=0.001\right)$ with cytokinaemia. Significant correlations between relative abundance of various genus from the Lachnospiraceae, Eggerthellaceae, Erysipelotrichaceae, Prevotellaceae, and Ruminococcaceae families $\left(r_{s}>0.400, \mathrm{p}<0.05\right)$ with EIGS markers were also observed. Associations between bacterial phyla, family, and genus were also observed for GIS, thermoregulatory, and physiological strain $\left(r_{s}>0.400, \mathrm{p}<0.05\right)$. Conclusions. - These landmark findings suggest that intestinal microbiota composition may contribute to the magnitude of EIGS. Further research is warranted to provide substantiation and justification for evidence-based intestinal microbiota altering nutritional interventions.

Supported by the Faculty of Medicine Nursing and Health Sciences and Sports Medicine Australia

The Impact of Post-Exercise Chocolate Flavoured Dairy Milk Beverage Consumption on Gastrointestinal Symptoms, Breath Hydrogen and Blood Glucose Responses in Endurance Athletes

Isabella Russo, MNutr ${ }^{1}$; Alan McCubbin, BNutr ${ }^{1}$; Judi Porter, $\mathrm{PhD}^{1,2}$; Louise Burke, $\mathrm{PhD}^{3}$; Ricardo J.S Costa, $\mathrm{PhD}^{1}$

${ }^{1}$ Monash University, Department of Nutrition \& Dietetics, Notting Hill, Victoria, Australia, ${ }^{2}$ Allied Health Clinical Research Office, Eastern Health, Box Hill, Victoria, Australia, ${ }^{3}$ Department of Sports Nutrition, Australian Institute of Sport, Bruce, ACT, Australia

Objective.-The study aimed to determine the impact of consuming a dairy milk beverage after prolonged strenuous exercise on gastrointestinal symptoms (GIS), breath hydrogen $\left(\mathrm{H}_{2}\right)$ and blood glucose responses. Methods.-Using a randomised crossover study design, 11 endurance male athletes consumed a chocolate flavoured dairy milk beverage (CM: $1.2 \mathrm{~g} / \mathrm{kg}$ carbohydrate and $0.4 \mathrm{~g} / \mathrm{kg}$ protein) or a carbohydrate-electrolyte beverage (CEB: isovolumetric with $0.76 \mathrm{~g} / \mathrm{kg}$ carbohydrate) after completing a $2 \mathrm{~h}$ glycogen depleting exercise protocol in temperate ambient conditions (20$\left.25^{\circ} \mathrm{C}, 40 \% \mathrm{RH}\right)$. The recovery beverage was provided in three equal boluses over a $30 \mathrm{~min}$ period. Assessment of gastrointestinal symptoms (GIS) and $\mathrm{H}_{2}$ in breath samples was undertaken pre-exercise and every 30min postexercise for $4 \mathrm{~h}$. Venous blood samples were collected pre-exercise and throughout recovery for analysis and determination of markers of intestinal injury (i.e., plasma I-FABP) and blood glucose responses. Results.-There were no differences in intestinal injury (mean (SD): 569 (751) pg/ml) in response to exercise between trials. During the recovery period, there were no trial differences in the severity of reported gut discomfort ( $\mathrm{p}=0.816$ ), total GIS ( $\mathrm{p}=0.668)$, upper GIS $(\mathrm{p}=0.593)$, lower GIS $(\mathrm{p}=0.460)$, or nausea $(\mathrm{p}=0.341)$. There was a main effect of time for breath $\mathrm{H}_{2}(\mathrm{p}=0.004)$, whereby a significant increase in breath $\mathrm{H}_{2}$ of clinical relevance (CM peak 19 (11) ppm, and CEB peak $19(17)$ ) was observed throughout recovery in both trials. Peak blood glucose response $(\mathrm{p}=0.014)$ and area under the curve $(\mathrm{p}=0.041)$ were greater during the $2 \mathrm{~h}$ after consumption of CEB $(7.8(1.1) \mathrm{mMol} / \mathrm{L}$ and 716 (66) $\mathrm{mMol} / \mathrm{L} / 2 \mathrm{~h}$, respectively) compared with $\mathrm{CM}(6.5(0.8) \mathrm{mMol} / \mathrm{L}$ and 660 (49) $\mathrm{mMol} / \mathrm{L} / 2 \mathrm{~h}$, respectively). Conclusions.—Compromised absorption during recovery on both trials is likely attributed to exercise-induced gastrointestinal syndrome, and has implications for nutrient bioavailability that warrants remediation. The slower glucose availability of CM is likely related to the greater protein and energy content.

Supported by Lion Dairy \& Drinks Co.

Epidemiology of Cycling Related Injuries on the Roads and Highways Treated at United States Emergency Departments Between 2009 and 2018

Morteza Khodaee, MD, MPH; Karin VanBaak, MD

Department of Family Medicine and Orthopedics, University of Colorado School of Medicine, USA

Objectives.-Traffic-related cycling injuries pose significant risks to athletes. In order to implement effective policies to decrease this risk, it is important to understand the epidemiology of such injuries. While there have been several studies on traffic related fatalities in this population, there is little in the literature about non-fatal traffic-related injuries seen in emergency departments (ED). Methods.-Data from the National Electronic Injury Surveillance System (NEISS) was used to evaluate all cycling-related ED visits from injuries that occurred on roads and highways from 20092018. Various outcome measures, including patient demographics, injury incidence and characteristics were examined. Results.-From 2009-2018, a total of 55,385 cases of traffic-related cycling injuries presented to the US EDs, an estimated average of 219,851 annual visits nationwide. The mean age of patients was $32 \pm 20$ years. There were more visits during the warm months of the year. About three-fourths of patients were male. The number of visits per year was stable over the 10 -year period. The most frequently injured part of the body was head and neck (32.4\%), upper extremity (29.0\%), and lower extremity $(21.5 \%)$. The highest frequency of injury type was contusion/abrasion $(26.1 \%)$, fracture $(22.1 \%)$, laceration $(12.6 \%)$, internal organ injury (12.3\%), and sprain/strain (10.6\%). There were 77 $(0.1 \%)$ fatalities and $6,571(11.9 \%)$ injuries that required hospitalization. Males had more fatalities than females $(0.2 \%$ vs $0.1 \% ; P<0.05)$. Conclusions.-This study shows a profile of traffic-related injuries in cyclists who present to the ED for medical care. The injury rate stayed unchanged, which may be concerning considering attempts to decrease these types of injuries by changing policies and legislations. 
Past Cold Injuries and Anatomical Regions of the Hand Where Cold Is Experienced by Search and Rescue Workers

Matthew D. White, $\mathrm{PhD}^{1}$; Elizabeth A. Gnatiuk, MKin ${ }^{1}$; Jacob M. T. Cartwright $^{1}$; Clara V. Etter, $\mathrm{MSc}^{1}$; Anthony. S. Perrotta, $\mathrm{PhD}^{1}$; Himani Boury $^{1}$; Emily J. Heaps, MBA ${ }^{2}$

${ }^{1}$ Laboratory for Exercise and Environmental Physiology, Department of Biomedical Physiology and Kinesiology, Simon Fraser University, Burnaby, BC, Canada, ${ }^{2}$ Wilson School of Design, Kwantlen Polytechnic University, Richmond, BC, Canada

Objective.-To help facilitate better design and production of cold weather mitts/gloves for ultra-athletes, Search and Rescue (SAR) workers and the military, the objective of this study was to assess in SAR workers the occurrence of cold injuries and where with their currently employed gloves and/or mitts their hands are experiencing cold. Methods.-Lions Bay Search and Rescue and North Shore Rescue workers participated in an online survey where they were asked 1) if they have been diagnosed with frostbite, Raynaud's Syndrome, cold immersion injuries or vibration white finger and 2) where their hands get cold during winter rescues. Respondents indicated in 33 anatomical regions on images of each hand where, including the anterior and posterior sides of all fingers, the thumb and the hand, they are experiencing cold. The study was approved by the SFU Office of Research Ethics. Results.-Twenty SAR workers from 25-64 years of age completed the survey, including 13 males, 5 females and 2 of unspecified sex. The males weighed (mean $\pm \mathrm{SD}$ ) $86.5 \pm 3.0 \mathrm{~kg}$, were 1.82 $\pm 0.07 \mathrm{~m}$ tall and had a body mass index (BMI) of $26.1 \pm 3.0 \mathrm{~kg} / \mathrm{m}^{2}$, whereas the females weighed $68.8 \pm 3.7 \mathrm{~kg}$, were $1.70 \pm 0.10 \mathrm{~m}$ tall and had a BMI of $22.2 \pm 3.0 \mathrm{~kg} / \mathrm{m}^{2}$; the unspecified sex group weighed $79.6 \pm 1.9 \mathrm{~kg}$, were $1.83 \pm 0.01 \mathrm{~m}$ tall and had a BMI of $23.6 \pm 1.9 \mathrm{~kg} / \mathrm{m}^{2}$. For the 20 respondents $15 \%$ had frostbite, $5 \%$ had Raynaud's Syndrome, $10 \%$ had cold immersion injury and $5 \%$ had vibration white finger. There were $45-70 \%$ of the respondents reporting cold for the finger and thumb distal phalanges whereas only $0-20 \%$ of the respondents reported cold on the palm of the hand and thenar eminence. Conclusion.-These preliminary results gave a low prevalence of cold injuries in this group and regions most often reported as cold are the distal phalanges of the fingers and thumb.

Supported by the Canadian Department of National Defense Innovation for Defense Excellence and Security (IDEaS) program and the Canadian Foundation for Innovation

\section{Limited Thermal Protection of Canadian Military Winter Garments in Extreme Cold Conditions}

Matthew D. White, $\mathrm{PhD}^{1}$; Elizabeth. A. Gnatiuk, MKin ${ }^{1}$; Jacob M. T. Cartwright ${ }^{1}$; Clara V. Etter, $\mathrm{MSc}^{1}$; Anthony S. Perrotta, $\mathrm{PhD}^{1}$; Himani Boury $^{1}$; Emily J. Heaps, MBA ${ }^{2}$

${ }^{1}$ Laboratory for Exercise and Environmental Physiology, Department of Biomedical Physiology and Kinesiology, Simon Fraser University, Burnaby, BC, Canada, ${ }^{2}$ Wilson School of Design, Kwantlen Polytechnic University, Richmond, BC, Canada

Objective.-To facilitate better design and production of cold weather mitts/gloves and boots for ultra-athletes, Search and Rescue (SAR) workers and the military, this study assessed the performance of the Canadian military winter kit in a single whole body extreme cold exposure in a climatic chamber. Methods.-A single male participant volunteered for the study who was 59 years of age, who weighed $74 \mathrm{~kg}$, who was 1.72 meters tall and had a body mass index of $25 \mathrm{~kg} / \mathrm{m}^{2}$. The participant at rest completed a single cold exposure to a temperature of $-60^{\circ} \mathrm{C}$. Instrumentation included the Canadian military winter garments to cover all exposed skin. Rectal core temperature $\left(\mathrm{T}_{\mathrm{RE}}\right)$ and skin temperatures $\left(\mathrm{T}_{\mathrm{SK}}\right)$ were measured with high precision thermistors. Skin temperature measurement sites included the nose, the ears and for each hand the thumbs, 1st and 4th finger, for each foot the 1st and 5th toe, the heels plus the instep and for each leg the knee as well as the lumbar region of the torso. Heart rate was measured with a sports computer and microclimate temperatures in the mitts were assessed with iButtons. The study was approved by the SFU Office of Research Ethics. Results.-Prior to entering the climatic chamber $\mathrm{T}_{\mathrm{RE}}$ was $37.6^{\circ} \mathrm{C}$ and $\mathrm{T}_{\mathrm{SK}}$ ranged from a low of $\sim 23^{\circ} \mathrm{C}$ on the feet to a high of $38^{\circ} \mathrm{C}$ on the nose. The decrease of $\mathrm{T}_{\mathrm{SK}}$ to the lower limit $10^{\circ} \mathrm{C}$ was in $\sim 17 \mathrm{~min}$ for the nose, in 18 to $30 \mathrm{~min}$ for the hands and feet whereas the ears, knees and lumbar $\mathrm{T}_{\mathrm{SK}}$ remained above $10^{\circ} \mathrm{C}$ throughout the exposure. Heart rate remained at $\sim 65 \pm 6$ beats/min during the cold exposure. Conclusion.-These preliminary results demonstrate the Canadian military winter kit mitts and boots provide limited duration thermal protection for a resting participant in extreme cold conditions.

Supported by the Canadian Department of National Defense Innovation for Defense Excellence and Security (IDEaS) program and the Canadian Foundation for Innovation

\section{Comparing the Energy Cost of Running With Two Different Back- pack Systems}

Volker Scheer, $\mathrm{MD}^{1,2,3}$; Solveig Vieluf, $\mathrm{PhD}^{3}$; Marc Schröder, $\mathrm{Bs}^{3}$; Pascal Lappe, $\mathrm{Bs}^{3}$; Hans-Christian Heitkamp, $\mathrm{MD}^{3}$

${ }^{1}$ Ultra Sports Science Foundation, Pierre Benite, France, ${ }^{2}$ Universidad a distancia de Madrid (UDIMA), University of Madrid, Spain, ${ }^{3}$ Institute of Sports Medicine, Department of Health, University of Paderborn, Germany

Objective.-Ultra-endurance running is a popular sport and runners often carry their own hydration and nutrition as additional weight in backpacks. A wide range of backpack systems exists. Our aim was to compare energy cost of running $(\mathrm{Cr})$ of two commercially available backpack designs, where weight is either equally distributed between the front and the back of the body (A) or the back alone (B). Methods.-Twelve male runners (mean age $22 \pm 1.7$ years) completed a standard exercise test on the treadmill $\left(\mathrm{VO}_{2} \max 52.2 \pm 4.7 \mathrm{ml} \mathrm{kg}^{-1} \cdot \mathrm{min}^{-1}\right)$ for determination of their individual lactate thresholds ((IAT) $2.95 \pm 0.38 \mathrm{mmol} / \mathrm{l}$, velocity (v) vIAT $12.2 \pm 1.3 \mathrm{~km} / \mathrm{h})$ and their individual testing speed (80\% vIAT $9.8 \pm 1.1 \mathrm{~km} /$ h). Further tests on the treadmill were conducted in randomized order for the measurements of ventilatory parameters during steady state conditions (at 5 and $20 \mathrm{~min}$ ) for calculation of $\mathrm{Cr}$ carrying backpack $\mathrm{A}$ and $\mathrm{B}$, weighing 3 and $6 \mathrm{~kg}$, respectively. Repeated measures ANOVAs were used, and significance was set at $\mathrm{p}<0.05$. Results. $-\mathrm{Cr}$ at $5 \mathrm{~min}$ with backpack $\mathrm{A}$ weighting $3 \mathrm{~kg}$ and $6 \mathrm{~kg}$ was $\left(1.02 \pm 0.14\right.$ and $\left.1.06 \pm 0.15 \mathrm{~J} \mathrm{~kg}^{-1} \mathrm{~m}^{-1}\right)$ and backpack B weighting $3 \mathrm{~kg}$ and $6 \mathrm{~kg}\left(1.03 \pm 0.15 \mathrm{vs} 1.07 \pm 0.16 \mathrm{~J} \mathrm{~kg}^{-1} \mathrm{~m}^{-1}\right)$. After 20 min of running $\mathrm{Cr}$ with backpack A weighting $3 \mathrm{~kg}$ and $6 \mathrm{~kg}$ was $\left(1.05 \pm 0.11 \mathrm{vs} 1.08 \pm 0.18 \mathrm{~J}^{\mathrm{kg}} \mathrm{kg}^{-1} \mathrm{~m}^{-1}\right)$ and backpack B weighting $3 \mathrm{~kg}$ and $6 \mathrm{~kg}\left(1.08 \pm 0.12\right.$ and $\left.1.13 \pm 0.13 \mathrm{~J} \mathrm{~kg}^{-1} \cdot \mathrm{m}^{-1}\right)$. Cr was lower for backpack A compared to backpack B after $20 \mathrm{~min}(\mathrm{p}=0.02)$. Conclusions. $-\mathrm{Cr}$ increased with loaded backpack running and over time. Cr was significantly lower for the front/back system compared to back only system after $20 \mathrm{~min}$, suggesting that during longer durations the equal distribution of weight is advantageous. 